\title{
MENTAL HEALTH SERVICES DURING THE PANDEMIC: THE EXPERIENCES OF LITHUANIAN PSYCHOLOGISTS
}

\author{
Rita Vaičekauskaitè \\ Klaipèda University, Lithuania \\ Jurgita Babarskienė \\ LCC International University, Lithuania \\ Jūratė Grubliauskienė \\ Klaipèda University, Lithuania
}

\begin{abstract}
With the COVID-19 pandemic and its restrictions, many countries face an unprecedented mental health crisis, which is being addressed in various ways, including the use of remote mental health services. Lithuania faced two quarantines: in March-June of 2020 and starting November 2020 up to Spring of 2021. The aim of this study is to explore the experiences of Lithuanian psychologists providing mental health services during the pandemic. Using the qualitative content analysis method, the following categories were made: from shock to discovery of new opportunities (differences in two lockdowns, better accessibility of services, help-seeking during the pandemic, and the importance of self-care) and contextual challenges (confidentiality, computer literacy, and blurred home/work boundaries). Implications for addressing psychological service issues are discussed, with an emphasis on self-care, setting boundaries, and finding new ways to enhance mental health via mediated communication as well as to reach out to vulnerable groups.
\end{abstract}

Keywords: COVID-19, disability, Lithuania, pandemic, psychological help, psychologists.

\section{Introduction}

Many people have directly experienced the major effects of the COVID-19 pandemic on their lives, and it is obvious that this effect will have long-term consequences in all spheres of life. But according to the experts, the pandemic has the biggest effect on the mental health (Giallonardo, et al., 2020; Fiorillo \& Gorwood, 2020). Mental health is mainly related to the capacity of a person to effectively deal with changes in his/her environment, which in the long-term perspective develops resilience. Mental health is more than the absence of mental disorders, following the definition of health by WHO that views health as a state of complete physical, mental, and social well-being and not merely the absence of disease. The research titled "What is Mental Health?" revealed that only $20 \%$ 
Vaičekauskaite et al., 2021. Mental Health Services During the Pandemic: the Experiences of Lithuanian Psychologists

choose the WHO (2001) definition as their preferred choice (Manwell et al., 2015). However, mental health has gained more meaningfulness and complexity during the pandemic, as recognized in the WHO concept. Moreover, the pandemic made mental health more evidently related with social factors. Certain health prevention behaviors, such as social distancing, are necessary to reduce the spread of the virus, but they make people feel frustrated, isolated, lonely, and worried about the future, which can increase stress, anxiety, and other disorders (Giallonardo et al., 2020). Thus, different support services, including psychological, are needed in learning to cope with stress in a health-friendly way.

WHO survey (2020) representing 130 countries reports that the problem of COVID-19 pandemic has disrupted or halted critical mental health services in $93 \%$ of the countries worldwide, while the demand for mental health continued to increase. Over $60 \%$ of respondents reported interruptions to mental health services are related to vulnerable people, including children and adolescents (72\%), older adults (70\%), and women requiring antenatal or postnatal services (61\%). In addition, $67 \%$ of participants saw disruptions to counselling and psychotherapy; 65\% believed it affected critical harm reduction services, etc.

While many countries (70\%) have adopted telemedicine or teletherapy to overcome hindrances to in-person services, there are significant disparities in the uptake of these interventions in high-income and low-income countries (WHO survey report, 2020). Therefore, WHO urges countries to monitor changes and disruptions in services so that they can address them as required. The objective of the current study is derived from the WHO's call to draw special attention to mental health services. This article focuses on the early pandemic experiences of psychologists who are providing mental health services. The aim of the current study is to conduct the qualitative analysis of the experiences of Lithuanian psychologists working during the COVID-19 pandemic.

\section{Literature Review}

The amount of the existing literature addressing mental health concerns related to the COVID-19 pandemic is rapidly growing. Preliminary evidence suggests that symptoms of anxiety and depression (16-28\%) and self-reported stress (8\%) are common psychological reactions to the COVID-19 pandemic and may be associated with disturbed sleep (Rajkumar, 2020). Some research note the risk of reactivation of previous traumas during certain events (Savarese, et al., 2020). This pandemic has been equated to such traumatic events as earthquakes and tsunamis, yet its impact goes beyond one specific place, as the threat of getting the disease is lurking in every person around us (Morganstein \& Ursano, 2020). This is especially true in the cities, where people experience more severe pandemic effects and much more uncertainty (Rubin \& Wessley, 2020). Studies 
related to SARS show that people, during that time, experienced helplessness, fear of getting sick and dying, feelings of blame, fear, and depression (Wu, et al., 2020). Yang \& Ma (2020) report that in China, with the onset of the COVID-19 pandemic, emotional well-being dropped as much as 74 percent. Women, young adults, people older than 60 are the most vulnerable in mental health outcomes during the lockdown (Jacques-Aviñó et al., 2020; Zhang \& Ma, 2020, Li et al., 2020).

The use of the online mode significantly changed the professional work of psychologists. The literature on COVID-19 shows that many people have experienced psychological suffering, and psychological support has proven to be important to encourage adaptation (Savarese et al., 2020). Online counseling has been the prevalent way to offer counseling services during the COVID-19 outbreak and encompasses services via the Internet, where the specialist and the client communicate utilizing computer-mediated communication. According to Situmorang (2020), we already have successful experience of distant counselling. Referring to previous studies (McKenna \& Bargh, 2000; Reynolds et al., 2006), we might find that counselees/clients who experienced uneasiness and social separation were more likely to create deeper connections through online/cyber counseling than through in-person counseling. Research has shown that counselees/clients have demonstrated both benefits and progress related to online counseling services (quoted in Situmorang, 2020).

Even though online work helped address the challenges of the COVID-19 pandemic, work from home affects mental health and family situation of the employees. It creates work-to-life and life-to-work conflicts, bigger work-related fatigue, a higher number of relational conflicts, poor general health as well as more interruptions and demands for an immediate response (Young, 2020; Palumbo, 2020). There is a thin line between professional and personal life which can lead to overworking, stress, and inability to balance work and leisure (Dragomir, 2020).

Research problem. Even though remote counseling is not a new phenomenon in psychology practice; however, its use was exceptional in counseling. Now, during the pandemic, psychologists must deal with the fact that remote counseling has become a daily practice. Moreover, people had no time to be prepared for this. Thus, it is a new challenge. Besides, there emerged an increased societal sensitivity to mental health issues because they are viewed as a disruption to psychological well-being and are not necessarily associated with the symptoms of the disease. Therefore, psychologists encounter a broader context of mental health issues. We suppose that lessons learned during the first quarantine helped to see new opportunities. Thus, current study's research questions are geared toward exploring the following: What new opportunities did psychologists 
Vaičekauskaite et al., 2021. Mental Health Services During the Pandemic: the Experiences of Lithuanian Psychologists

find? What new opportunities did the pandemic allow to discover for the clients? How, in the point of view of psychologists, did the societal attitude change toward mental health problems?

\section{Method}

Participants. This research included 10 participants, 9 females and one male (average age of 30 years) from Lithuania. The participants were recruited using convenience sampling and were asked to participate in an online interview or answer questions in written form. The participants consisted of health and clinical psychologists working in the Lithuanian healthcare system, such as mental health centers, hospitals, and rehabilitation centers. The data was collected from May to December 2020.

Process of collecting data. The interview was conducted using social media. The interviews took from $30 \mathrm{~min}$. to an hour, were voice recorded and transcribed. Some participants responded to the questions in the written form. To safeguard participant confidentiality, participant number was used instead of their name (P1 stands for participant 1 ).

Interview questions. The study was carried out by using semi-structured interviews. The participants were asked to describe their experiences of providing mental health services during the COVID-19 pandemic. The core interview questions were: What is unique about your personal experience of psychological services during the pandemic? What are your experiences of working online during the pandemic as opposed to the usual face-to-face work before the quarantine? What are the key mental health issues you face in counselling during the pandemic?

Transcription, coding, and analysis. Interviews were transcribed verbatim or used the written responses of the participants and analyzed by the means of qualitative content analysis (Graneheim, 2017). Through the process of decontextualization, the researchers coded the interviews individually, then reviewed the codes and, alongside with recontextualization, came up with seven common subcategories, from which two major categories were derived (Fig.1). They are discussed in detail in the Results' section.

\section{Results}

The current study findings encompass 2 categories and 7 subcategories (Fig. 1): 


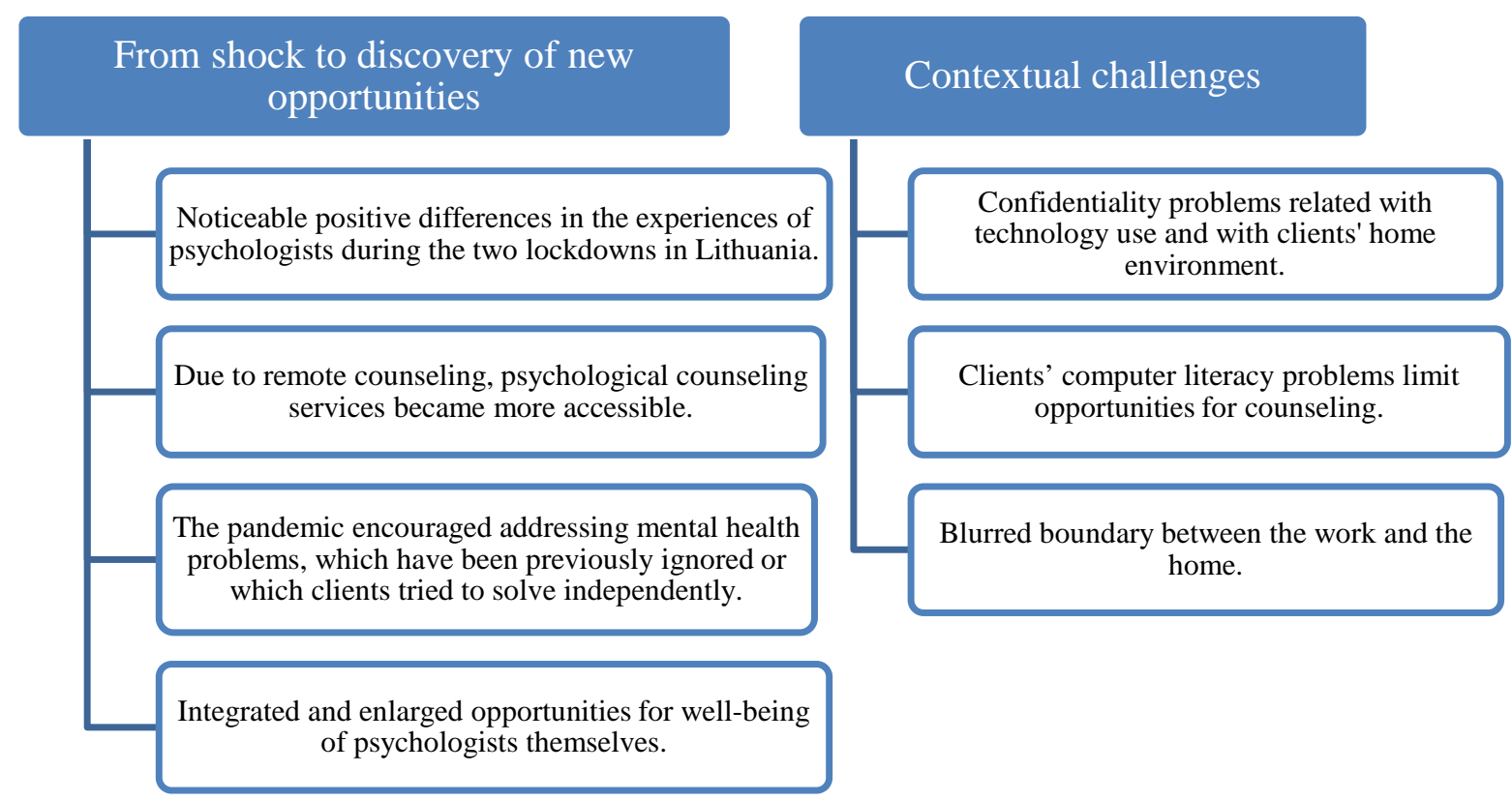

Figure 1 The System of Key Subcategories and Categories

The category From shock to discovery of new opportunities addresses four key ideas dealing with the experiences of psychologists and mental health issues. Each is described below, and key ideas are indicated by participant quotes.

Noticeable positive differences in the experiences of psychologists during the two lockdowns in Lithuania. The first quarantine for the participants was more of a shock, when everything seemingly stopped, while the second time they were more prepared: In the first quarantine, it was like...take a vacation and everyone was frozen, and everything stopped (P1).

We were all scared in the spring because everything was very negative; we were all going to die. We were looking for positive information. Isolation from people was deadly (P2).

We did not have video and audio tools for quite a while, so we could only do counseling by phone. Also, not all patients comply with safety and hygiene requirements $(P 3)$.

Furthermore, numbers in services differed: March showed a significantly reduced number of services provided (P7).

In March, only remote services were provided, while now mixed services are provided: partly face to face, partly remotely (P8).

During this [November] quarantine, we work and have contact, not just remotely ( $P$ 9).

The work is happening normally now [during the second quarantine], as before the first quarantine. The same number of patients, mobilized, protected, protective measures, strict requirements. Only the administration gives more 
Vaičekauskaite et al., 2021. Mental Health Services During the Pandemic: the Experiences of Lithuanian Psychologists

work remotely. But we are working with patients directly, do [COVID-19] tests weekly, and patients are counselled... only after receiving negative test (P1).

Part of the services are provided remotely: using video and the telephone. Such services were not available before the quarantine (P4).

For psychologists, more preparation was needed to transition to remote work in addition to counseling online being more intense and emotionally tiring: The assessment process is more difficult. It was necessary to remake the methodologies, take pictures, but everything was on the computer (P2).

It seems to me that, as I do counseling remotely, I communicate more intensely, I get more tired as if my senses are more strained (P10).

When working remotely, if a significant number of customers are available during the day - there is greater emotional fatigue (P8).

Psychologists note that both psychologists and the clients transitioned to the second quarantine quite smoothly: At the moment, it is easier to plan consultation times (remotely), and customers are more easily accepting of remote counseling services (P4).

During quarantine, both in the spring and now, I work from home, remotely. I have more individual online consultations and lectures in zoom; weekly work meetings and meetings take place. In March, I prepared more articles, leaflets, did more communication with colleagues by email (P5).

The biggest difference was the redirection of all work to remote work. (P6).

So far, I can't name [the difference between the two quarantines], the work has moved more into the remote mode, but the work goes smoothly, and there is a lot of work (P8).

To summarize, transitioning to remote ways of providing mental health services was challenging, but smooth, with the second quarantine allowing more flexibility and providing new opportunities because the lessons that were dictated by the challenge were well-learned between the quarantines.

Due to remote counseling, psychological counseling services became more accessible. Yet online nature of communication helped some people to finally seek counseling, lay down their defenses, open up more, and be more aware of themselves: It seems to me that during the current quarantine, when the difficulties arise, people are more courageous to seek help (P5).

It was scary at first. In online groups, the group was more open on the Internet, more relaxed. Maybe some were without cameras, but they spoke, wept, broke down, and then they turned cameras on, got support, there was less defensiveness and rejection. Maybe because that was online? No longer a need to escape. Clients were afraid but would still talk. Some of them would not have been able to stay in a real face-to-face group, but they did online. Once you overcome, you see yourself, can watch yourself, there is a better sight of emotions in the face, micro reactions $(\mathrm{P} 3)$. 
In terms of quality, [online counseling] is not poorer, it is even easier for loved ones to open up; they think less about talking and about shame related to the child's problem and can concentrate on the problem...in remote counseling the client does not accept the problem as accusation and assumes more responsibility, while face- to-face they feel the need to defend themselves more. So, there is more collaboration (P1).

For some of those who are seeking remote consultations, they are more convenient, providing a sense of security (P7).

Some clients miss sessions due to COVID-related fears or circumstances: More clients do not come to counseling sessions because of the need to selfisolate, because they are afraid of face-to-face contact, because they cannot travel to another municipality, thus more counseling is being done remotely. But the nature of help is still primarily the same (P9).

To conclude, working remotely meant more challenges, such as missing nonverbal cues, technological and access issues, and confidentiality problems, but online work also helped some clients to seek help because some long-term issues accumulated and were exacerbated by the pandemic restrictions.

The pandemic encouraged addressing mental health problems, which have been previously ignored or which clients tried to solve independently. On the other hand, quarantine made the issues more severe and may have been the turning point in helping some people seek help: The issues of those who had difficulties before the quarantine became even more difficult. 'I cannot do anything, I don't know anymore, I'm apathetic, depressed.' Probably issues were exacerbated by that isolation, for those who have mental problems, they became even more pronounced (P2).

Quarantine brought out those problems that people already had. In the past, we were hiding [issues] until we could handle them, but then there was a turning point, what was hidden there, came out. Quarantine pushed to search for help (P4).

The pandemic makes isolation greater; people do not have the ability to exercise, communicate with their peers, the problem of loneliness. More people want to communicate with a psychologist. Teenagers do not want to communicate with loved ones. Study motivation has fallen. Teachers are tired. It's extremely hard (P1).

Clients come due to various mental issues: Because of the problems that had been there before the pandemic: anxiety, depression, interpersonal relationships, suicidality, etc. Only a proportion of those coming for help are related to pandemic experiences (e.g., loss of work, difficulties in relationships, loneliness) (P6). 
Vaičekauskaite et al., 2021. Mental Health Services During the Pandemic: the Experiences of Lithuanian Psychologists

Client problems are anxiety, internal tension, stress, fears for the future (feeling of insecurity, fear of losing their jobs, downtime, etc.), disagreements in families or among couples (P5).

Anxiety, fear, stress, strained relationships between couples/families (P3). Interpersonal relationships (conflicts, anger, tension), loneliness (desire to "talk to someone"), difficulties in parenting (P7).

We work with all patients. More sleep disturbances due to burnout; children spend more time at the computer, go to bed late, burnout, insufficient sleep (P1).

Characteristic difficulties associated with emotions, stress, relationships, loss of work/ learning or finances, loss, increase in addiction, increase in the risk of suicide, exacerbation of symptoms of mental disorders. During the pandemic, anxiety-related themes are more pronounced (P2).

The psychologists report that pandemic is the most challenging for the young, single people, the elderly and those with mental health problems, including those who care for people with disabilities: The most severely pandemic is experienced by lonely young people, persons with mental disorders, people living with people with mental disorders or those caring for disabled loved ones. It is associated with strict quarantine restrictions (P8).

Other people who come for counseling during the pandemic are: Dependent persons. Patients with chronic diseases. Those caring for a patient. Parents raising young children. Young adults experiencing an interpersonal relationship crisis. Problems: fear, anxiety, tension, stress, poorer sleep associated with COVID-19, loneliness, apathy, problems of interpersonal relationships, anger (P10).

In addition, persons experiencing family relationship crises, most pressing relationship problems, divorce, psychological trauma, children's learning difficulties at a distance, work problems, financial problems (P8). Also, children from 11 years of age (their parents apply for counselling for children) and adults. Women are the main ones (P5).

People with disabilities or those caring for them also come for counseling, but the differences in numbers before and during the pandemic are not significant: I had one client- the mother of a boy with Asperger's syndrome. But significant difference [in numbers of clients with a disability] was observed (P5). During quarantine, people with disabilities and chronic diseases sought help. In my experience, there were more people with disabilities, who sought help, but there were also close family members too (P5).

In summary, key mental health issues became more difficult because of isolation and uncertainty, and some types of clients were especially vulnerable during the pandemic. In order to help clients, psychologists needed to take better care of their own well-being, as described in the next subcategory. 
Integrated and enlarged opportunities for well-being of psychologists themselves. Still, what is rewarding during the pandemic is that participants are able to self-care more: What gives joy is that I have more time for myself. I can jog, walk, draw, walk by the sea with a dog, do self-relaxation (P2). They are also able to get feedback from clients (in the workspace), do favorite activities, engage in physical activity, read books, articles (P7).

Not only that, but also take care of yourself: rest, time for yourself, physical activity, communication, however, remotely with loved ones (P6).

I have rituals, walk outdoors after work, do meditation, listen to lectures and communicate with loved ones (P1).

I can sleep longer, take time to do the work because there is no need to go anywhere. Walk outdoors. Communication with loved ones at least with the help of technology. Reading books and magazines. Delicious homemade food (P6).

Finally, the most important thing is to maintain relationships with significant people in our lives, to invest in relations during the pandemic. (P10).

To summarize, participants reported more opportunities for self-care and appreciated more time for family and leisure activities amidst the business of their work.

The Contextual challenges category encompasses issues related to computer literacy, confidentiality and home-work balancing issues. The subcategories are described and illustrated with participants quotes below.

Confidentiality problems related with technology use and with clients' home environment. On the other hand, remote counseling is characterized by more interruptions and confidentiality problems: Boring work slightly complicates contact, for example, frequent disturbances, such as family members, which during the consultation interfere with the client (P10).

There are additional issues related to ensuring the confidentiality of the clients when the client is unable to have a safe space at home- there are family members around during consultation (P9).

There are additional problems with data security (P5).

Contact remotely decreases because some clients prefer to communicate without a camera. This complicates communication between the psychologist and the client, it is difficult to use certain therapeutic tools, to respond to customer reactions, to experience emotions during counseling (P2).

Clients may also expect the counselor to be available immediately and do other things during online sessions: Help is often expected here and now, even without prior registration. More clients miss the counseling sessions (P7).

During group sessions, clients tend to do their other work and avoid switching on cameras or microphones. It is impossible to use certain techniques, especially with children. It is difficult to ensure a safe, confidential environment 
Vaičekauskaite et al., 2021. Mental Health Services During the Pandemic: the Experiences of Lithuanian Psychologists

for the client. However, psychological counselling services have become more attractive and accessible to young people and working people (P2).

Clients' computer literacy problems limit opportunities for counseling. The problems with remote work have to do with computer literacy, loss of some nonverbal-cues, difficulty in establishing rapport, and data security issues. For example, the elderly struggle with technology: Elderly clients needed help with technical difficulties: how to activate the app or camera, adjust the sound. Also, it is unusual to see clients in their home environment, where personal belongings are visible (P10).

Telephone counselling is more difficult: invisible non-verbal language; some information is lost. Nor are all people able to use or have computers, which prevents remote video consultation. It may be more difficult to establish and maintain contact (P5).

Sometimes there is less involvement of customers in the session; it is more difficult to read the body language of clients (P8).

Blurred boundary between the work and the home. The participants reported both the benefits and the drawbacks of working remotely. First, there is no need to travel, unless one has contact hours and work in the office; thus, they can save on time and fuel, and clothes: You don't need to ride, the number of errands is reduced, there is no need to waste time, to drive, all you need to do is to connect to the computer (P4).

On the other hand, they also note that it is difficult to maintain healthy boundaries between what is work and what is home: Boundaries between rest and work. It is difficult to adjust the time, relax, to retreat from domestic work because I am always in the same room (P2).

In addition, the number of hours has increased due to irregularity of the hours and being on call even during days off or weekends: When working from home and with dramatically increased workloads, the boundary between work and "home" disappeared. Workloads require a lot of work after work and weekends. People are overworked (P8).

\section{Discussion}

The present study explored the experiences of Lithuanian psychologists working during the COVID-19 pandemic. The results show a shift to remote work due to the pressing mental health needs. The first quarantine was perceived as a big shock; however, with time, psychologists transitioned to online work. This is in line with the literature that discusses the need for quality remote services to the most vulnerable groups of people, such as children, women, young adults, the elderly, people with disabilities, and the lonely people (Jacques-Aviñó, et al., 2020; Zhang \& Ma, 2020, Li et al., 2020). Despite the drawbacks of technological 
skills and the lack of nonverbal cues, online counseling in pandemic circumstances was viewed as beneficial. Moreover, the problems related to online counseling during the pandemic are similar to earlier identified ones (Situmorang, 2020). Pandemic significantly changed psychologists' and clients' point of view towards remote counseling; namely, it is supposed to be seen as a new possibility for the future without the pandemic. Challenges of online mental health services are related to the boundary between work and home environment being blurry either for specialist or clients (Young, 2020; Palumbo, 2020; Dragomir, 2020).

Pandemic experience of psychologists informs about changing societal attitudes toward mental health as an integral part of well-being. During the pandemic, people realized the importance of social factors for mental health. They mainly attributed their increased anxiety and stress to the changed social circumstances: limited direct social contacts, lack of mobility, and distance while communicating. People associated both, the emergence of new mental health problems and the deepening of the existing ones, with the above-mentioned social factors. Due to the pandemic, mental health is less pathologized and less personalized. People started prioritizing their mental well-being as an important part of staying healthy. Social contacts and environment have gained added value as the experience of isolation people find out as stressful and hard to manage by themselves.

\section{Conclusions}

At this time, it is still too early to draw conceptual conclusions about the phenomenon studied. As a result, our conclusions are only initial insights trying to understand the phenomenon of remote psychological counseling.

COVID-19 pandemic has been an unprecedented crisis in many spheres, including mental health. Nevertheless, psychologists and their clients were able to go through the transition, embracing the remote means of mental healthcare. Due to quarantine restrictions, many mental health issues came into the open, thus psychological help has become even more pertinent. On the other hand, access to such care is still complicated for some vulnerable groups who lack computer literacy. The current study emphasizes such critical factors as self-care, setting boundaries between work and home, and making better use of technologies to reach vulnerable target group clients. The COVID-19 crisis is not over yet, and the need for mental health workers will continue to increase.

The pandemic has altered certain attitudes toward counseling by both psychologists and clients. Remote means of counseling which were initially viewed as a barrier eventually started to be perceived as a new opportunity. During the time of the pandemic, there has been a noticeable change in societal 
Vaičekauskaite et al., 2021. Mental Health Services During the Pandemic: the Experiences of Lithuanian Psychologists

attitudes toward mental health as an integral part of well-being; therefore, people seek help even in those cases when they would not have hurried to do so before.

\section{Acknowledgements}

This research is funded by the European Social fund according to the activity 'improvement of researchers' qualification by implementing world-class R\&D projects’ of measure No. 09.3.3-lmt-k-712.

\section{References}

Dragomir, L.-M. (2020). The Balance between Personal and Professional Life in the "Work from Home" Era. Annals of the Constantin Brancusi University of Targu Jiu-Letters \& Social Sciences Series, 2, 41-48. https://doi.org/10.1080/25741292.2020.1863560

Fiorillo, A., \& Gorwood, P. (2020). The consequences of the COVID-19 pandemic on mental health and implications for clinical practice. European Psychiatry: the Journal of the Association of European Psychiatrists, 63(1), e32. https://doi.org/10.1192/j.eurpsy. 2020.35

Giallonardo, V., Sampogna, G., Del Vecchio, V., Luciano, M., Albert, U., Carmassi, C., Carrà, G., Cirulli, F., ... \& Fiorillo, A. (2020). The Impact of Quarantine and Physical Distancing Following COVID-19 on Mental Health: Study Protocol of a Multicentric Italian Population Trial. Frontiers in psychiatry, 11, 533. https://doi.org/ 10.3389/fpsyt.2020.00533

Graneheim, U., Lindgren, B. M., \& Lundman, B. (2017). Methodological Challenges in Qualitative Content Analysis: A Discussion Paper. Nurse Education Today, 56, 29-34. https://doi.org/10.1016/j.nedt.2017.06.002

Jacques-Aviñó, C., López-Jiménez, T., Medina-Perucha, L., De Bont, J., Gonçalves, A. Q., Duarte-Salles, T., \& Berenguera, A. (2020). Gender-based approach on the social impact and mental health in Spain during COVID-19 lockdown: a cross-sectional study. BMJ open, 10(11), e044617. http://dx.doi.org/10.1136/bmjopen-2020-044617

Li, Z., Ge, J., Yang, M., Feng, J., Qiao, M., Jiang, R., ... \& Yang, C. (2020). Vicarious traumatization in the general public, members, and non-members of medical teams aiding in COVID-19 control. Brain, Behavior, and Immunity, 88, 916-919.https://doi.org/ 10.1016/j.bbi.2020.03.007

Manwell, L. A., Barbic, S. P., Roberts, K., Durisko, Z., Lee, C., Ware, E., \& McKenzie, K. (2015). What is mental health? Evidence towards a new definition from a mixed methods multidisciplinary international survey. BMJ open, 5(6). https://doi.org/10.1136/bmjopen2014-007079

Morganstein, J. C., \& Ursano, R. J. (2020). Ecological disasters and mental health: causes, consequences, and interventions. Frontiers in Psychiatry 11(1). https://doi.org/ 10.3389/fpsyt.2020.00001

Palumbo, R. (2020). Let me go to the office! An investigation into the side effects of working from home on work-life balance. International Journal of Public Sector Management, 33(6/7), 771-790. https://doi-org.libproxy.lcc.lt/10.1108/IJPSM-06-2020-0150 
Proto, E., \& Quintana-Domeque, C. (2021). COVID-19 and mental health deterioration by ethnicity and gender in the UK, PLoS One, 16(1), e0244419. https://doi.org/ 10.1371/journal.pone.0244419

Rajkumar, R. P. (2020). COVID-19 and mental health: A review of the existing literature. Asian Journal of Psychiatry, 52, 102066. https://doi.org/10.1016/j.ajp.2020.102066

Rubin, G.J., \& Wessely, S. (2020). The psychological effects of quarantining a city. BMJ, 368, m313. https://doi.org/10.1136/bmj.m313

Savarese, G., Curcio, L., D'Elia, D., Fasano, O., \& Pecoraro, N. (2020). Online University counselling services and psychological problems among Italian students in lockdown due to Covid-19. Healthcare (Basel, Switzerland), 8(4), 440. https://doi.org/10.3390/ healthcare8040440

Situmorang, D. D. B. (2020). Online/cyber counseling services in the COVID-19 outbreak: Are they really new? The Journal of Pastoral Care \& Counseling, 74(3), 166-174. http://doi.org/10.1177/1542305020948170

WHO. (2020). WHO Survey COVID-19 disrupting mental health services in most countries. Retrieved from https://www.who.int/news/item/05-10-2020-covid-19-disrupting-mentalhealth-services-in-most-countries-who-survey

Wu, K. K., Chan, S. K., \& Ma, T. M. (2005). Posttraumatic stress, anxiety, and depression in survivors of severe acute respiratory syndrome (SARS). Journal of Traumatic Stress: Official Publication of The International Society for Traumatic Stress Studies, 18(1), 3942. http://doi.org/10.1002/jts.20004

Yang, H., \& Ma, J. (2020). How an epidemic outbreak impacts happiness: Factors that worsen (vs. protect) emotional well-being during the coronavirus pandemic. Psychiatry research, 289, 113045. https://doi-org.libproxy.lcc.lt/10.1016/j.psychres.2020.113045

Young, E. (2021). Challenging boundaries of work space. The Psychologist, 18-19. Retrieved from https://thepsychologist.bps.org.uk/volume-34/january-2021/challenging-bounda ries-work-space

Zhang, Y., \& Ma, Z. F. (2020). Impact of the COVID-19 pandemic on mental health and quality of life among local residents in Liaoning Province, China: A cross-sectional study. International journal of environmental research and public health, 17(7), 2381. https://doi.org/10.3390/ijerph17072381 